regular dental follow-up visits. These findings indicate that guidelines on prophylaxis are not being followed adequately.

In summary, the study highlights the poor prognosis of IE and identifies opportunities for improvements in its prevention and management.

Original article Tornos P et al. (2005) Infective endocarditis in Europe: lessons from the Euro Heart Survey. Heart 91: 571-575

\section{Atherosclerosis-like lesions of the aortic valve in unselected adults}

Researchers from Finland have discovered that atherosclerosis-like lesions in the aortic valve are common in adulthood, even among young subjects with no history of atherosclerotic vascular disease.

Kuusisto et al. examined the aortic valves of 48 unselected necroscopy subjects aged 2040 years $(n=15), 41-60$ years $(n=17)$, or 61 years and older $(n=16)$. A clinical history of cardiovascular risk factors was uncommon among the younger subjects, all of whom died in accidents or by suicide. In the middle-aged and elderly groups, however, a history of coronary artery disease and cardiovascular risk factors was prevalent, and acute myocardial infarction was the cause of death in 13 of these individuals.

At necroscopy, macroscopic calcification of the aortic valve was found in only two subjects, and there were no cases of aortic stenosis. However, the pathologists identified an atherosclerosis-like subendothelial thickening above the elastic lamina on the aortic side of at least one of the valve leaflets in 45 subjects (94\%). Moreover, all subjects in the oldest age group had a lesion in every valve leaflet. The mean lesion area was significantly associated with age, ranging from $0.1-0.2 \mathrm{~mm}^{2}$ in younger subjects, $0.5-0.8 \mathrm{~mm}^{2}$ in the middle-aged group, to $1.3-2.3 \mathrm{~mm}^{2}$ in those aged $\geq 61$ years.

In conclusion, atherosclerosis-like lesions of the aortic valve were prevalent among these Finnish adults and occurred in the absence of macrocalcification. In their discussion, Kuusisto and colleagues relate these findings to the development of aortic stenosis, and suggest that this disease process might begin in early adulthood.

Original article Kuusisto J et al. (2005) Atherosclerosis-like lesions of the aortic valve are common in adults of all ages: a necroscopy study. Heart 91: 576-582

\section{Small-vessel coronary artery disease: bare-metal stents versus balloon angioplasty}

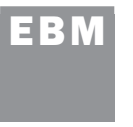

Lesions in small coronary arteries account for up to half of all coronary stenoses, but it is unclear whether stenting or percutaneous transluminal coronary angioplasty (PTCA) represents the best reperfusion strategy in this setting. In their recent meta-analysis, Agostoni et al. have compared the relative merits of these two approaches.

The investigators searched the literature (from 1994 to 2004) for prospective randomized trials comparing bare-metal stenting and angioplasty for the treatment of lesions in coronary arteries of less than $3 \mathrm{~mm}$ diameter. Eight published studies were included in the subsequent analysis. Five trials that had been published only as abstracts were also included. Following data abstraction from all 13 studies, the team compared the risk of death, myocardial infarction, repeat revascularization and major adverse cardiovascular events in patients treated with stenting vs PTCA.

In total, 4,383 patients were randomized in the 13 studies, either to PTCA $(n=2,097)$ or stenting $(n=2,286)$ and the median duration of follow-up was 8 months. The pooled results indicated that major adverse cardiovascular events were significantly less common among patients who received stents than among those who underwent angioplasty (17.6\% vs $22.7 \%$, odds ratio $0.71,95 \% \mathrm{Cl} 0.57-0.90)$. This difference was largely driven by a lower rate of repeat revascularizations in the stenting group, and did not apply when suboptimal angioplasty was excluded from the analysis. There were no significant differences between the two reperfusion methods in terms of the risk of death or myocardial infarction. Statistical heterogeneity was present in the overall analysis, but this disappeared when the subgroup of optimal PTCA was analyzed separately.

The authors conclude that stenting showed a moderate advantage over angioplasty, and that optimal balloon angioplasty with provisional stenting might be equally as effective as routine stenting.

Original article Agostoni P et al. (2005) Is bare-metal stenting superior to balloon angioplasty for small vessel coronary artery disease? Evidence from a meta-analysis of randomized trials. Eur Heart J 26: 881-889 